\title{
Using Case-based Reasoning for Situation Classification on Campus Violence
}

\author{
Nuka Nwiabu \\ Int. Science and Technology Initiatives \\ Massachusetts Institute of Technology \\ Cambridge, USA
}

\author{
Jackson Akpojaro \\ Department of Mathematics and Computer Science \\ College of Natural and Applied Sciences \\ Western Delta University, Oghara, Nigeria
}

\begin{abstract}
Controlling crimes in institutions of higher learning requires the ability of an in-depth analysis of the current situation. For understanding of the current situation and deducing consequences for taking actions, higher-level reasoning about the situation has to take place. In this paper, an approach for situation awareness for cult activities is presented. The approach uses case-based reasoning to predict the evolution of the current violence and to select the appropriate action. Casebased enables prior experiences to be utilized in the task of situation assessment.
\end{abstract}

\section{General Terms}

Case-based reasoning, situation awareness

\section{Keywords}

Campus violence, cult groups, law enforcement agencies, prior knowledge, university administrator

\section{INTRODUCTION}

Campus violence, a complex and widespread issue, has received significant attention from the public, university administrators, and law enforcement agencies. The wide range of acts that fall under this rubric include all violent behavior and threats of violence, as well as any conduct that can result in injury, damage property, induce a sense of fear, and otherwise impede the normal course of academic work [13]. Threats, harassment, intimidation, bullying, stalking, intimate partner violence, physical or sexual assaults, homicides, and gangsters fall within this category [1]. The prevalent situation on Nigeria university campuses is gangsters otherwise known as cultism. Cult groups are groups of students whose activities are shrouded in secrecy and which could use unconventional and unacceptable means in dispensing its activities, which are mostly esoteric and individualistic. These groups now abound in Nigerian Universities and there seems to be no signs of their ever stopping as there is no approach at the moment on how to check the activities of these groups. Many higher institutions of learning throughout Nigeria have instituted programs to help prevent cultism on campuses. These efforts can go a long way toward mitigating the threat of such occurrences. However, no method of studying actions of administrators and cult groups for predicting campus cultism exists. First, it is critical to understand that campus cult violence does not happen at random or "out of the blue." Rather, a group usually displays some behaviors of concern in response to administrative policy or actions of opposing groups. The awareness of these indicators and the subsequent implementation of an action plan to mitigate potentially violent form are essential components of campus safety.
It is not sufficient to use information from a single source to determine the action of a cult group. There is need for a higher-level assessment which allows to infer additional information from diverse sources through an evaluation of relations between objects of campus events as well as to incorporate background knowledge.

This paper presents a work that fused data from various sources, converts them into a more abstract symbolic representation and then interprets the situation using prior knowledge through case-based reasoning (CBR). This higherlevel assessment consists of a situation interpretation and a decision making part. The situation interpretation is used to get a deeper understanding of the current situation and the output serves as a basis for the decision making process which involves balancing of different actions according to their projected consequences. An action is assessed by predicting potential progressions of the situation.

\section{BACKGROUND}

In safety critical scenarios, like air traffic control in the aviation industry, anesthesiology in healthcare, terrain monitoring in military command and control, university campuses, maintaining situation awareness of the environment is essential for effective decision-making. Situation awareness (SA) is a cognitive process in decision making and is defined as "the perception of elements in the environment within a volume of time and space, the comprehension of their meaning, and the projection of their status in the near future" [3]. The Endsley situation awareness model has three layers comprising perception, comprehension, and projection. The perception layer recognizes all the necessary information about the environment. The comprehension layer interprets the perceived information in order to understand the current state of the environment. The projection layer uses knowledge of the current state of the environment to predict its future state. Representations of domain knowledge for situation awareness are stored in longterm memory (mental model or schema) [3]. The level of SA that an operator has is dependent on the complexity of the available mental model. As an operator becomes more experienced with the domain, their mental model becomes more developed, which explains why experts are better at integrating multiple cues compared to novices [6]. The difference between the expert and the novice in their level of $\mathrm{SA}$ is experience-based reasoning. One of such reasoning methods is case-based reasoning (CBR).

Human cognition in situation awareness and decision making can be categorized as rule-based, case-based or any others [12]. In some situations, a set of rules is worked out in order 
to select an action. In other situations experiences are often stored as cases and "remembering" is done by comparison with preserved cases. Case-based reasoning is a psychological theory of human cognition that addresses issues in memory, learning, planning, and problem solving [15]. The psychological assumption of the case-based reasoning paradigm is that memory is predominantly episodic and so it is richly indexed such that experiences are related to each other in many complex and abstract ways. CBR builds on an understanding on how humans assess situations [14], supporting recognition primed decision (RPD) framework proposed by Gary Klein [14]. The framework emphasizes the role of experiences in human decision making processes during time critical situations. Klein pointed out that humans depend more on past experience rather than deliberate rational analysis of possible alternatives during time critical decision making. For example, when the general domain knowledge is difficult to extract and instead requires reasoning based on local knowledge or where it is difficult to formulate rules describing the situations [5]. CBR also helps in situations of incomplete domain data [12]. Case-based reasoning (CBR) is one of the most effective paradigms of knowledge-based systems [10]. Reasoning by humans is done by recalling memories guided by experiences of their immediate environment. CBR draws from experiences of past cases in order to solve new problems. Operators interpret and understand new situations in terms of prior experiences [15] and preserve the new experience by retaining it in memory.

Feng et al [4] used rules in a computational model of situation awareness for exploiting goal-based contextual information to achieve user specific situation awareness. Agents, one for each individual operator, communicate with the situation model and extract information of relevance for presentation to their respective users in accordance to the user context. Defining necessary heuristics based on bounded definition of the domain (command and control) and responding to each and every new development was difficult due to Feng's rulebased decision support engine [4].

Rule based systems require a careful procedure in order to ensure the consistency of the rule-base. A set of rules is worked out in order to understand the situation. Background knowledge is given implicitly in the rules and the order of the rules. Rule-based systems are not able to work with experiences [5] and rules are created by a limited number of experts. Their knowledge and ignorance are implicitly reflected in the rules [7]. Unlike the experience-based systems, the only way to explain a decision in rule-based systems is to report the chain of inferences. Experience-based systems such as case-based systems contain more explicit knowledge which can be used to enrich the explanation of a decision and thus making it more intuitive. Case-based systems have several advantages compared to classical rulebased systems. It facilitates better maintainability and expandability than rule based systems [14] since new knowledge is added by integrating new cases automatically to the case-base. Partial matching is another advantage of casebased systems. Even if a case does not match exactly, it can still be considered for problem solving [12].

Kofod-Petersen et al [9] used case-based reasoning in modeling SA in an ambient intelligent system. The "perception" and the "awareness" layers of the system are comparable to Endsley's perception and comprehension layers of situation awareness. The third (sensitivity) layer adapts the ambient system's behavior to the current situation. The sensitivity layer does not anticipate future situations to make it a projection layer. The adaptation of the system to the current situation was possible by combining a user's context with environmental elements at the perception level.

Vacek et al [18] used case-based reasoning in a computational model of situation awareness for autonomous driving. CBR was used to interpret the current situation and selecting the appropriate behavior. Future situation behaviors were known by their projected consequences using the expectation value. The expectation value is calculated for each applicable behavior and the behavior with the highest overall value is selected as the behavior of the future situation. Ting et al [17] also applied features of expectations during the projection stage while working on the use of CBR to build a computational SA model for military operation in urban terrain (MOUT) simulations. The approach uses violation of expectations to determine behaviors or actions. Violation of expectation in the approach is categorized as invariant and variant. Invariant expectations must be fulfilled or else there is danger while the violation of variant expectations is merely an alert of possible threats. The system of both Vacek [18] and Ting [17] rely only on cues from the environment without considering the user's context.

While existing systems on campus violence prevention document and wait for a concrete observation of a direct threat, most often perpetrators of violence provide no direct threat; written or verbal [8]. Therefore, the reliance on the emergence of direct threats weakens the ability to detect early threats. As a solution, Denkle [2] proposed that universities must implement threat assessment systems responsible for detecting, assessing, and monitoring students of concern. Smith [16] proposed a combination of approaches beginning with the admissions process and continuing through an ongoing assessment process, which includes the use of a central reporting system. The central reporting system maintains comprehensive documentation of any behavioral patterns with risk indicators; and the system responsible for making threat assessments continues ongoing monitoring of those persons of interest based on prior knowledge.

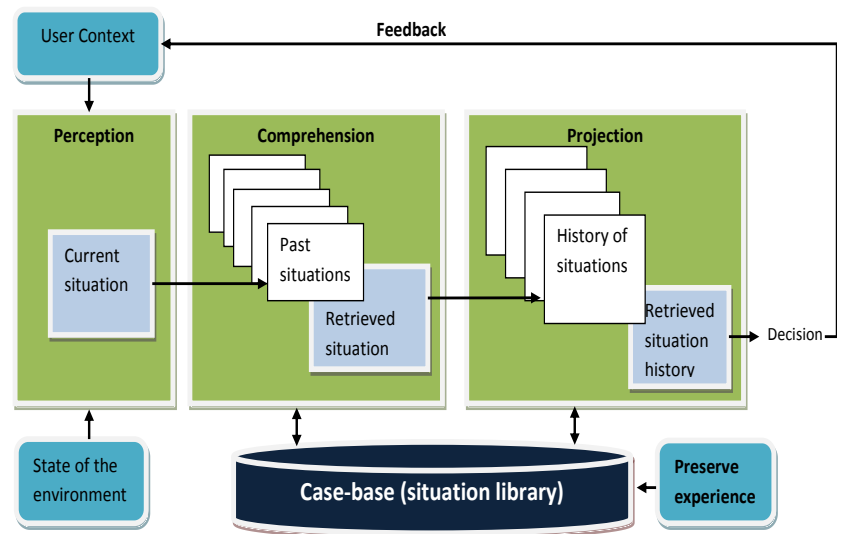

Figure 1: Case-Based Situation Awareness Model [11]

\section{CAMPUS VIOLENCE PREVENTION USING CASE-BASED SITUATION}

To design a system to provide a university administrator good situation awareness that will bring about good decision making, a couple of components are necessary. As shown in 
Figure 1, the system consists of seven main components: User context, State of the environment, Case-base (situation library), Perception, Comprehension, Projection, and Preserve experience.

Context in this work defines the goal, expectation and the specific needs of the operator. State of the environment collects cues of the current situation and sends the information to the perception component. The perception component delivers data in terms of predefined objects from the context of users and the environment and converts this data into an abstraction in order to feed it into the reasoning process. Comprehension is the retrieval component which extracts all situations of the case-base that have the highest similarity with the current situation. The Projection component is where existing knowledge is exploited by a reuse process to identify consequences of the current situation on future situations and present actions that are most suitable to avert the situations. The operator carries out decision making by selecting the appropriate action. The judgment of the operator on the future of a situation is also used to direct further perception of the system through feedback. The last component is the preserve phase which is applied after the selected action is implemented and found to be workable. A newly acquired experience is entered into the case-base in order to update the knowledge base.

The interpretation of the current situation consists of steps depicted in the "Comprehension" level of Figure 1. Each step manages campus event graph which includes all objects together with their attributes and relationships. The output is the most suitable action which is executed by the operator.

In the second step, the quantitative data from the perception is mapped to a qualitative description because symbolic information is more feasible for reasoning processes and reduces the amount of data [18]. A situation consists of an event on campus, all objects in this event, an estimation of the actions of other groups and the mission goal of the administrator among others.

The task of preventing an impending crisis situation is solved by applying the case-based reasoning paradigm. The basic idea of case-based reasoning is to try to solve a problem by remembering previous situations which are similar to the given one and reuse the solution that was used in that situation. In a campus situation interpretation, it means: given an impending violence, what is the appropriate action that should be taken? Try to remember previous situations which were similar to the current one, understand, how and why a specific action was taken, and transfer the solution to the current situation. Additionally, remember the new experience by integrating the new case into the case-base. The following is the stage by stage discussion of the design.

\subsection{Situation Library}

Using the case-based reasoning process, the first step is to define a situation followed by situation or case-base construction in a campus violence scenario.

\subsubsection{Definition of a Situation}

The definition of a case is a crucial part for case-based reasoning. The definition of a situation has consequences for finding similar situations compared to the current situation on the one hand and on the other hand it influences the retrieval of problem solving knowledge which has to be used in a given situation.

A campus administrator has several goals in a university and therefore it is not quite clear where a case starts and where it ends. For this reason fine granular distributed situation are used (See Table 1).

Table 1. Sample actions

\begin{tabular}{|l|l|}
\hline \multicolumn{1}{|c|}{ Group Behavior } & \multicolumn{1}{c|}{ Administrative Response } \\
\hline $\begin{array}{l}\text { Intimidating, harassing action } \\
\text { And verbal threats }\end{array}$ & Suspension of student \\
\hline Bring weapon to campus & Notify the police for arrest \\
\hline $\begin{array}{l}\text { Statement indicating approval } \\
\text { of campus violence to resolve } \\
\text { a problem }\end{array}$ & Keeping a close watch \\
\hline $\begin{array}{l}\text { Statements indicating } \\
\text { Desperation over academic } \\
\text { problems }\end{array}$ & Silence \\
\hline $\begin{array}{l}\text { Direct threats of harm } \\
\text { Drug abuse }\end{array}$ & $\begin{array}{l}\text { Notify the police and the state } \\
\text { Security service }\end{array}$ \\
\hline $\begin{array}{l}\text { Extreme changes in normal } \\
\text { behavior }\end{array}$ & Room to room search \\
\hline $\begin{array}{l}\text { Numerous conflict with } \\
\text { Fellow students }\end{array}$ & $\begin{array}{l}\text { Evaluate and determine potential } \\
\text { causes in change of behavior }\end{array}$ \\
\hline $\begin{array}{l}\text { Victims should report to the Dean } \\
\text { of students affair and then, the }\end{array}$ \\
\hline $\begin{array}{l}\text { Clandestine meetings } \\
\text { Administrative policy }\end{array}$ & Notify campus police \\
\hline $\begin{array}{l}\text { Influx of students from other } \\
\text { institutions into the campus }\end{array}$ & Holding of seminars \\
\hline Boycott of lecture & Monitoring \\
\hline $\begin{array}{l}\text { Confrontation with opposing } \\
\text { group }\end{array}$ & Silence \\
\hline
\end{tabular}

A situation resulting from actions as shown in Table 1 represents a snapshot of the situation rather than a time interval. A time interval is represented by a concatenation of situations. The core components of a situation are (1) the description of the event (2) the action of the administrator (3) the actions of cult groups and (4) an assessment of the represented situation according to different measurements.

The description of the event is done using the knowledge modeling. The event is characterized by a set of instantiated concepts and relations. The instances represent objects or parts of the event. A-boxes are used in order to describe the axiomatic and factual knowledge of the event in description logic. Relations are used to describe connections between objects as well as states of single objects.

The second component of a situation is the current plan of the administrator, in terms of action to be executed. Additionally, how the action will be executed is stored. The actions of the cult groups are stored as well. These actions are not known and are therefore estimated.

The last part of a situation is the assessment, which enables one to compare different situations and find out which one the most desirable is or the other way round, which situations should be avoided. To carry out similarity assessment, a single value $p$ between 0 and 1 is calculated to express the assessment of the situation, where a higher value expresses a 
more appropriate action. The assessment is based on the evaluation of different features, whereas a feature can only be fulfilled or not. Each feature belongs to one of four classes:

a) Academic disturbance: This class contains all features that mark actions that affect normal academic program on campus, like disturbing students in the library and class rooms.

b) Disobedience: This class represents all features that classify a violation of a university rule but do not constitute a dangerous situation, for example, boycott of classes by a group.

c) Critical situation: All features contained in this class are indicating situations, where a significant violence exists. This can be accomplished through many different factors like disturbing students in the library and class rooms, boycott of classes or if unforeseeable things happen like protest during examination period.

d) Tragic: The last class consists of features that characterize situations where violence did happen. These are for example, clashes between cult groups that led to lose of lives, violence resulting to destruction of properties.

A function $f\left(x_{i}\right)$ assigns a value between $[0,1]$ to $x_{i}$ (where $x_{i}$ denotes fulfilled features, $i=1,2, \ldots, n$ ). This means that the more critical a feature, the lower its value. In general, the situation assessment is defined as;

$$
p=\min \left\{f\left(x_{i}\right)\right\}, \quad i=1,2, \ldots, n
$$

The consequences of taking the minimum of $p$ is that only the most important fulfilled feature counts and all less important features are ignored regardless of how many apply.

\subsubsection{Situation Library Construction}

The construction of the situation library relies on the definition of a situation. The main focus lies on indexing of the situations in order to alleviate and speed-up the search for the most similar situations. The indexing scheme is based on links between different situations and facilitates the search for situations by walking through the library. Situations are linked in three different dimensions. In the first dimension, situations are organized hierarchically according to the specialization of the situation. In the second dimension, situations at the same level of specialization share a link representing their differences. And lastly, links denote temporal evolutions of situations.

The hierarchical arrangement represents an order of situations from the most general to the most specific situation. Specialization takes place either because a concept or a role is more specific or new instances of concepts or roles are added to the situation. In doing so, the link holds the reasons that led to the specialization of that situation, i.e. it contains all the differences which make this situation a more specific one. This index is used later at the situation retrieval stage in order to extract the most similar situations. In order to provide an entry point into the library, a top element is used which is more general than any other situation.

The second type of link interrelates to the situations at the same level of specialization. The edge between two situations holds the difference between these two situations. These links are used for generalization of new situations. The linkage of situations based on temporal interrelation helps in predicting the consequences of the execution of different actions and thus selecting the right action for the administrator. A situation is linked temporally with another, if its contents have changed significantly over time and is a direct evolution of the preceding situation. The applied action which leads to the temporally succeeding situation is stored together with the link. Because of the applicability of different actions, a situation can have multiple succeeding situations. Additionally, a probability of occurrence is saved.

If an action is applied for a given situation, there is no need that there exists exactly one temporal successor rather than multiple successors. This accommodates the fact, that the prediction of how the situation will evolve is always tainted with uncertainty. Therefore, each applied action for a given situation is assigned multiple temporally succeeding situations, each succeeding situation together with its probability of occurrence. The different actions of the cult groups are not stored in the temporal linkage itself, but rather given by different situations in the library. For the prediction of the evolvement of the situation, it is assumed that cult groups keep their current behavior.

Figure 2 shows how one action leads to different succeeding situations. In this example, action A1 can result in three succeeding situations 2,3 and 4 . For each successor, the probability of occurrence is denoted. Another specialty is that it is also possible that different actions share succeeding situations. In the given example, for both action A1 and A2, one successor is situation 4 .

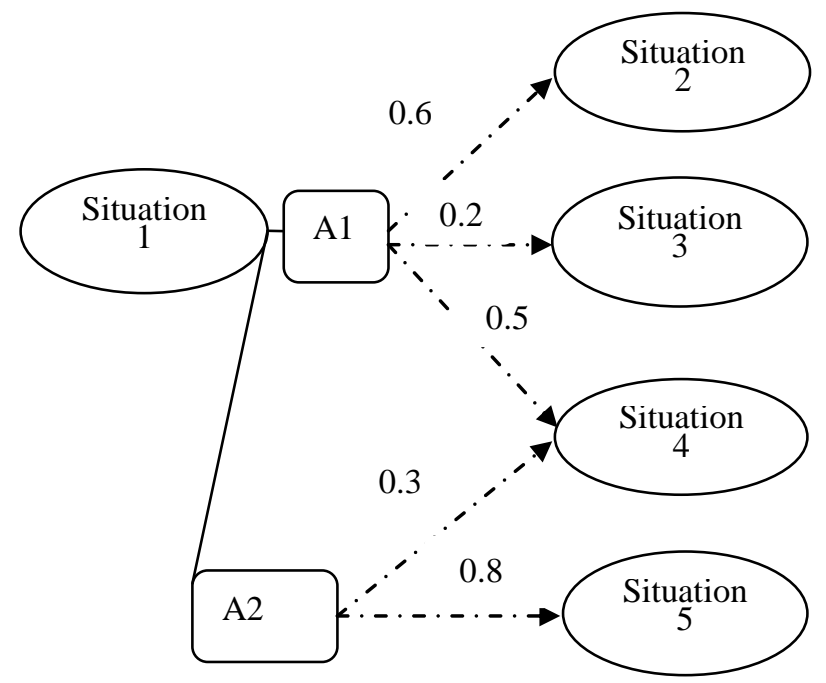

Figure 2: Temporal linkage of situations using different actions.

\subsection{Perception}

The recognition of the status and the dynamics of relevant elements in the environment is the first stage in determining situation awareness. The elements are the entities. Entities are objects in the environment which have attributes. Events are problems defined by the environment and context. An event injection causes the case-based situation awareness model to reassess the relevant entities attributes and their relation with each other which eventually will result in new situation awareness. This layer recognizes the state of the campus with information from multiple sources and the university 
administrator context and then structures the information into a coherent shape.

\subsection{Comprehension (Situation Retrieval)}

Situation retrieval is the phase which extracts the most similar situation in the library for the given situation. The best situations are searched by traversing the library recursively along the paths given by the hierarchical organization. In the following, each directly linked specialization of a situation will be called a child node of that situation. A situation matches the current situation if it is completely contained in the current situation. Instances and relations of the situation can be generalized to fulfill the matching, e.g. a situation described as a "Disobedience situation" matches a situation which is recognized as a "Crisis situation."

Beginning with the top element, a child node is visited if it matches the current situations. This is done for all child nodes of a visited node. If a node has no matching child nodes, a best situation is found and added to the set of retrieved situations. The termination of the retrieval is guaranteed, since the situations are organized in a cyclic, directed graph. Single situations from the library can be used multiple times because of different mappings of the individual situations. Situations can be seen as some sort of template and the partial matching uses different elements of the event in order to fill the placeholders of the template.

The result is a set of situations which covers parts of the current situation. For each extracted situation, the individual situations and actions, which were used for pattern matching, are noticed.

\subsection{Projection (Situation Reuse)}

The purpose of the phase reuse of situations is to analyze existing knowledge contained in the retrieved situations and to generate a solution from this knowledge. In campus violence prevention domain, the goal is to select the appropriate action for the university administrator. For this, different applicable actions are evaluated hypothetically and the most appropriate action is selected as the best suitable solution.

In order to clarify the procedure, we first assume, that only one matching situation was found in the situation retrieval phase. Going by the modeling of the library, the situations are linked both hierarchically and temporally. Links represent the temporal evolution of the situation. To select the most appropriate action, all possible evolutions of the situation are regarded by analyzing the temporal successors of the retrieved situation. Then, to detect critical situations at an early stage, the prediction can consider multiple levels of successors. This can be done by combining the assessment along the prediction path using the minimum. The uncertainty of the prediction increases with the length of the prediction path.

In order to assess the temporally succeeding situations, different rates for each situation are evaluated. The probability of occurrence of each situation and the overall assessment value is given by the expectation value which indicates the applicability of the action. The higher the expectation value, the better the action is applicable. The expectation value is derived for each applicable action. The action with the highest overall value is taken (See Figure 3).

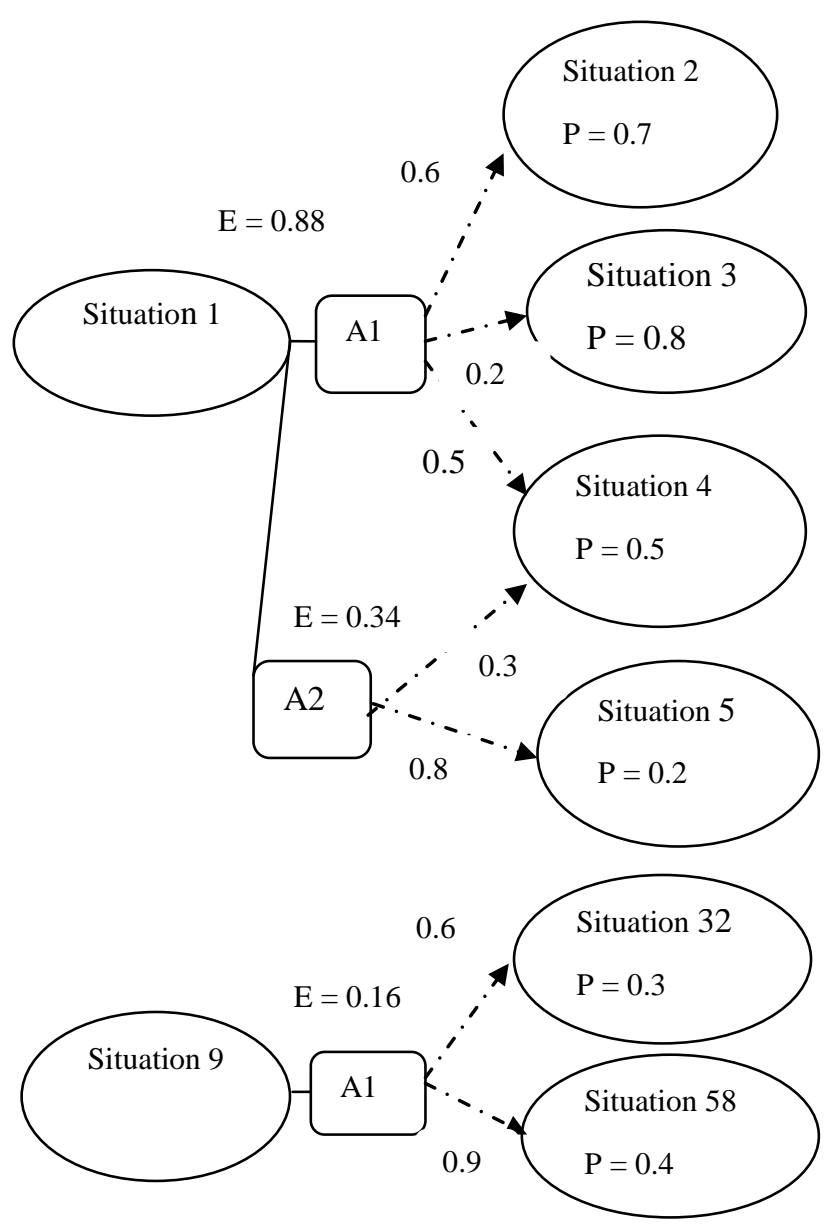

Figure 3: Choosing the best action given multiple situations.

In Figure 3, it is assumed that situation 1 is the only retrieved situation and two different actions, A1 and A2 can be taken. The overall rating $\mathrm{p}$ of each situation, together with the probabilities of occurrence, gives the expectation value of 0.88 for action $\mathrm{A} 1$ and a value of 0.34 for action A2. Thus, action $\mathrm{A} 1$ is taken.

In a case where multiple situations are extracted, the procedure is as follows: First, for each extracted situation, an assessment value is assigned for each action of that situation. After that, for each action the minimum over all situations, which offer that action, is calculated and this minimum is regarded as the rate of applicability of that action. Finally, the action with the highest rating is taken.

An example of multiple situations is given in Figure 3. Here, the expectation values for all actions in all situations are calculated. This led to action A1 in situation 1. But because both situations share action A1, the overall minimum of that action is A1 from situation 9 and therefore, action A2 from situation 1 with an assessment value of 0.36 is taken.

\subsection{Retaining Situations}

The last phase is used to preserve newly acquired experience and to provide it for future use. This phase is executed after the implementation of the action and the applicability of the action is known. 
In the "reuse" stage, different situations were extracted representing the most similar situations and the best suitable action was generated based on these situations. After implementation, the next sets of situations with best similarity are extracted according to the situation "retrieval" phase. From this selection, it is possible to check, which temporally succeeding situations from the time of implementation are really happening. With this information, the probability of occurrence can now be updated for all these situations.

If for a best situation none of the temporally succeeding situations did happen, a new situation must be created and integrated into the library through the following steps: (1) Specify all objects of the current situation that are part of the previous situation and the matching situation and all new appeared objects. (2) Make the current situation conform to all these objects and their relations. (3) The objects should be generalized to the level of the matching situation.

The newly created situation can then be integrated into the case-base. This implies adding the situation to the case-base and creating all links for this situation. A generalization of situations in the case-base happens, if the branching factor of a situation is higher than a certain value. In that case, all situations at the same level as the added situation are taken into account. Generalization is done by identifying the similarities between the new situation and an arbitrary situation at the same level of specialization. These two situations are replaced by this new generalized situation and added as child nodes.

\section{DISCUSSIONS OF RESULTS}

The study investigates the number of accurate predictions of the system with past violence threats in a Nigeria university campus. It also assesses the similarity between the system's recommendations and the expert solutions. Five persons from university administration and five persons from the university security unit working on campus violence participated in the experiment. Two independent variables were system types and system accuracy. System accuracy is a factor to represent how accurately the system provides SA and actions based on an entered query. A query is entered by subjects into the system to see the degree of similarity. To estimate how accurate these predictions are, we used the 10-fold crossvalidation technique to evaluate the methods. The case-base contains forty five (45) past situations. Five test datasets are taken out of the case base and matched against forty train cases in each round of the evaluation. The result in Table 2 provides a mean accuracy of 0.8 for the system, which implies that out of every 10 predictions eight are correct.

Table 2. Mean Accuracy

\begin{tabular}{|l|l|l|l|l|l|}
\hline Evaluations & 1 & 2 & 3 & 4 & 5 \\
\hline Accuracy & 0.81 & 0.85 & 0.83 & 0.78 & 0.81 \\
\hline
\end{tabular}

\begin{tabular}{|l|l|l|l|l|l|}
\hline Evaluations & 6 & 7 & 8 & 9 & 10 \\
\hline Accuracy & 0.89 & 0.86 & 0.76 & 0.84 & 0.82 \\
\hline
\end{tabular}

The "revise" stage is a manual adaptation level which requires additional human reasoning and increased participation of the experts in evaluating the recommended actions. In this qualitative evaluation, situation $20,8,12,5$ and 14 were used as test cases. Except situation 5, solutions of situations retrieved by the system as best matches were the same with selected best solutions by experts (See Table 3 ).

Table 3: Similarity Assessment

\begin{tabular}{|l|l|l|l|l|l|}
\hline Test Case & 20 & 8 & 12 & 5 & 14 \\
\hline System's Best case & 13 & 36 & 27 & 16 & 25 \\
\hline Expert's Best case & 13 & 36 & 27 & 9 & 25 \\
\hline
\end{tabular}

In some situations, administrators did not properly document past experiences. The system limitation is that, it is not able to estimate the consequences of actions when no situation knowledge exists.

\section{CONCLUSION}

This work, campus violence prevention is implemented using a previous architecture (Case-based Situation awareness) [11]. The current situation is interpreted by comparing the current situation with past experiences on campus. An action is taken based on a prediction of the evolution of the current situation. Newly acquired knowledge is preserved as experience in the library.

Administrators were not able to document all campus experiences. This resulted in few preserved situations. This system can only recommend actions to situations with prior experiences. Where no similar past situation is found, the system is handicapped.

Our future work will use CBR and rules to investigate how to estimate the consequences of actions when no situation knowledge exists. Actions for situations of more general relationships shall be represented in the explicit form of rules.

\section{REFERENCES}

[1] Booth, B., Vecchi, G. M., Hasselt, V. B., and Romano, S. J. 2005. Captive-Taking Incidents in the Context of Workplace Violence: Descriptive Analysis and Case Examples. Aggression and Violent Behavior: A Review Journal 10: 533-551.

[2] Denkle, J. H. 2009. Introduction to the Contemporary Update. New Directions for Student Services: 128.

[3] Endsley, M. R. 1995. Toward a Theory of Situation Awareness in Dynamic Systems. Human Factors and Ergonomics Society, 37:32-64.

[4] Feng, Y., Teng, T., and Tan, A. 2009. Modeling Situation Awareness for Context-aware decision Support. Expert Systems with Applications: 455-463.

[5] Gupta, M., and Mukherjee, S. 2009. Towards Situation Awareness in Integrated Air Defence using Clustering and Case-based Reasoning. In Pattern Recognition and Machine Intelligence third International Conference.

[6] Hoogendoorn, M., Lambalgen, R. M., and Treur, J. 2011. Modeling Situation Awareness in Human-like Agents 
using Mental Models. In Twenty-Second International Conference on Artificial Intelligence.

[7] Jones, R. E., Connors, E. S., Mossey, M. E., Hyatt, J. R., Hansen, N. J., and Endsley, M. R. 2010. Modeling Situation Awareness for Army Infantry Platoon Leaders using Fuzzy Cognitive Mapping Techniques. In Conference on Behavior Representation in Modeling and Simulation.

[8] Keller, E. W., Hughes, S., and Hertz, G. 2011. A Model for Assessment and Mitigation of Threats on the College Campus. Journal of Education Administration, 49(1), 7694.

[9] Kofod-Petersen, A., and A. Aamodt, A. 2009. Casebased Reasoning for Situation-aware Ambient Intelligent: A Hospital ward Evaluation Study. In LNAI 5650, pp. 450-464.

[10] Liao, S. 2000. Case-based Decision Support System: Architecture for Simulating Military Command and Control. European Journal of Operational Research, 123:558-567.

[11] Nwiabu, N. D., Allison, I., Holt, P., Lowit, P., and Oyeneyin, B. 2012. Case-based Situation Awareness. In IEEE International Conference on Cognitive Methods in Situation awareness and Decision Support, New Orleans, LA, pp. 22-29.
[12] Richter, M. M. 1998. Case-based Reasoning Technology: From Foundation to Application, volume 1400, Chapter 1, pp. 1-15. Springer, Berlin/ Heidelberg.

[13] Rugala, E. A., and Romano, S.J. 2008. Workplace Violence: Mind-Set of Awareness. Spokane, WA-Center for Personal Protection and Safety.

[14] Salem, A. M. 2007. Case-based Reasoning Technology for Medical Diagnosis. World Academy of Science, Engineering and Technology.

[15] S. Slade, S. 1991. Case-based Reasoning: A Research Paradigm. AI Magazine, 12(1):42-55.

[16] Smith, H. 2011. Violence on Campus: Practical Recommendations for Legal Educators. Retrieved from University of Tennessee: http://works.bepress.com/sandra_thomas/16/.

[17] Ting, S., Zhou, S., and Hu, N. 2010. A Computational Model of Situation Awareness for mouth Simulation. In International Conference on Cyber worlds.

[18] Vacek, S., Gindele, T., Zollner, J. M., and Dillmann, R 2007. Situation Classification for Cognitive Automobiles using Case-based Reasoning. In IEEE Intelligent Vehicle Symposium, Istanbul, Turkey. 